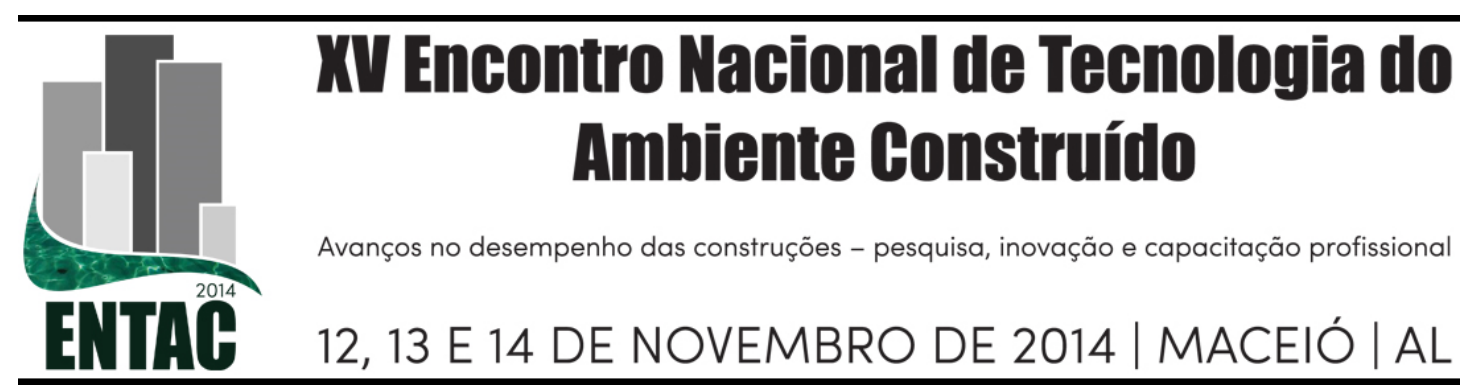

\title{
PLATAFORMA BIM COMO SISTEMA DE GESTÃO E COORDENAÇÃO DE PROJETO DA RESERVA CAMARÁ
}

\section{LEÃO DE LIMA, Alexandre César(1); SILVA DE ALBUQUERQUE, Daniela Maria (2); DE LIMA PEREIRA, Ingrid Kellen (3); MELHADO, Sílvio (4)}

(1) Universidade de Pernambuco, e-mail: acll_pec@poli.br (2) Universidade de Pernambuco, e-mail: dmsa_pec@poli.br, (3) Universidade de Pernambuco, e-mail: iklp_pec@poli.br (4) Universidade de São Paulo,e-mail: silvio.melhado@poli.usp.br

\begin{abstract}
RESUMO
A coordenação de projetos é um grande desafio. Trabalhar a coordenação de projetos em meio a implementação de um novo modelo de desenho e planejamento torna-se ainda um desafio maior. Esse artigo tem como objetivo fazer uma análise crítica da gestão de projetos através do uso da plataforma BIM de um empreendimento de grande porte na região metropolitana do Recife chamado Reserva Camará. Pretende-se mostrar dentro de um cenário em transformação, de mudança de modelo de trabalho, quais foram os desafios encontrados, que benefícios e dificuldades foram surgindo ao longo do caminho, que lições aprendidas pelos participantes do processo de forma a contribuir para a formatação de um fluxo de trabalho futuro ideal. Para tal, foi feita uma revisão bibliográfica, uma entrevista com a equipe de coordenação de projetos e finalmente uma análise do processo de implantação da plataforma BIM. Como o empreendimento ainda se encontra em fase de fundação, não foi possível apresentar um resultado definitivo que contemplasse todas as etapas do projeto, desde o planejamento até a execução, entretanto, é esperado pela equipe de coordenação de projetos que haja uma redução de custo e tempo de execução de obra significativa em função de se estar corrigindo falhas projetuais que só seriam vistas na etapa de execução. Por outro lado, faz-se necessário dizer que a forma como foi concebido o fluxo de trabalho e atividades de transferência dos projetos em CAD para BIM pela equipe de coordenação de projetos não condiz com o processo ideal de utilização do BIM. Essa prática precisa ser repensada dentro de um modelo colaborativo onde todos os projetos já teriam que ser desenhados na plataforma BIM e não como hoje está sendo realizado.
\end{abstract}

Palavras-chave: Building Information Modeling (BIM), modelo de coordenação de projetos, escritório de coordenação de projetos, Computer-aided design $\mathrm{CAD}$.

\begin{abstract}
Project coordination is a big challenge. Performing project coordination within the implementation of a new design model and planning becomes yet a bigger challenge. This article aims to do a project management critical analysis through the implementation and use of BIM platform of a huge building complex in the metropolitan area of Recife called Reserva Camara. The idea is to show along a transforming and working process changing scenery, which challenges have been found and what benefits and difficulties have been faced along the way; what were the lessons learned by the stakeholders in order to contribute to improve future working experiences. In order to do so, the following procedures have taken place: a literature review, an interview with the project coordination team and finally an analysis of the implementation process of BIM platform. As the work was still in its foundation phase, it was not possible to present a definite result including all project stages, from the conception to the implementation, however, it is expected by the project coordination team to have a drastic reduction on the cost and time during the implementation phase as a result of project inconsistences found and already corrected, which would only be seen during this phase. On the other hand, it's important to say that the
\end{abstract}


way the working process was conceived and its design transferring activities under CAD platform to BIM platform by the project coordination team is not in accordance with the ideal BIM process. This practice needs to be evaluated within a collaborative model where all project designs should be done under the BIM platform not as it is being done currently.

Keywords: Building Information Modeling (BIM), Project Coordination model, project coordination team, $C A D$.

\section{INTRODUÇÃO}

Nos dias atuais, o mercado de trabalho e o ambiente de negócios está cada vez mais competitivo. Com a globalização, o uso de soluções projetuais e as tecnologias de softwares que utilizam a tecnologia Building Information Modeling (BIM), tem ganhado espaço não apenas no desenvolvimento de projetos de engenharia e arquitetura, como também em áreas de gestão e gerenciamento de projetos.

Essa prática tem interferido diretamente na funcionalidade, desempenho e desenvolvimento de soluções prévias com a finalidade de prever e evitar problemas futuros que ocasionam custos diretos e indiretos no desenvolvimento de uma obra, podendo também evitar custos humanos como acidentes de trabalho.

A indústria da Construção Civil tem mudado seu panorama incorporando, além dos critérios de sustentabilidade, novas tecnologias a fim de renovar sistemas de gerenciamento e gestão desde a fase da construção propriamente dita como na reavaliação dos conceitos e otimização dos procedimentos que norteiam todo o ciclo de vida de um processo.

A metodologia de gestão que vem sendo aplicada ao projeto consolida o que foi destacado por Oliveira (2005):

[...] Os processos de concepção e projeto são estratégicos para a qualidade do edifício ao longo do seu ciclo de vida. E a busca de novos métodos e processo que possam considerar precocemente a totalidade das questões envolvidas no projeto é de extrema relevância para o sucesso dos empreendimentos e para o progresso do setor da construção.

No entanto, a prática demonstra que a falta de conhecimento ainda é uma de muitas barreiras que precisam ser transpostas para a integração dessa tecnologia na melhoria do processo de projetos, não apenas no Brasil, mas em diferentes países e cenários. (MANZIONE, 2013).

Neste sentido, a escolha do projeto Reserva Camará permite a identificação desse procedimento uma vez que é pioneira na região a implantar a tecnologia BIM para a conformação do empreendimento.

Dessa forma, pretende-se nesse artigo, abordar o estudo da tecnologia BIM aplicada ao novo projeto do Complexo intitulado Reserva Camará, localizado na região Metropolitana do Recife, no Município de Camaragibe, a fim de identificar as vantagens e desvantagens de implantação da tecnologia BIM na gestão de projetos e elucidar os diversos aspectos da importância do seu uso para a melhoria da qualidade, execução e gestão de projetos, bem como a importância que o mercado tem dado para tal tecnologia.

\section{METODOLOGIA}

Para a elaboração da pesquisa foram realizados primeiramente levantamentos bibliográficos, selecionando artigos científicos, monografias, vídeos, sites para obter informações sobre a tecnologia BIM, compreensão do tema e contextualização. 
Após esta etapa foram feitas buscas de estudos de casos para o aprofundamento das pesquisas realizadas, a fim de obtermos maior compreenção das formas práticas de aplicação da tecnologia BIM e seus pontos positivos e negativos numa obra, sendo definido o empreendimento Camará como caso prático de aplicação desse instrumento. O processo de coleta de informações na empresa para o artigo se passou com a visita ao escritório de coordenação de projetos. Nesta etapa foram realizadas entrevistas com a equipe de coordenação de projetos da Reserva Camará, que são em número de 04 (quatro) pessoas, através da elaboração de questionários e gravações de áudio. Por fim, concluiu-se a pesquisa com a elaboração deste artigo consolidando todas as informações coletadas.

\section{APLICAÇÃO DO BIM NA GESTÃO E COORDENAÇÃO DE PROJETOS}

\subsection{Conceitos e aplicações}

De acordo com Eastman et al. (2008, p.13), BIM é "uma tecnologia de modelagem e um grupo associado de processos para produção, comunicação e análise do modelo de construção", e segundo esse conceito, envolve tecnologias e processos que devem ser usados na produção, comunicação e análise dos modelos de construção.

A Tecnologia BIM "é um processo de geração e manutenção dos dados da construção durante todo o seu ciclo de vida (projeto, construção e operação)". Em geral, utiliza-se softwares de modelagem dinâmica, tridimensional, e em tempo real para aumentar a produtividade no projeto e na execução da obra. As informações contidas neste modelo abrangem a geometria da construção, as relações espaciais, as informações geográficas e as quantidades e propriedades dos elementos utilizados na construção.

Diante disso, um dos questionamento que se faz relaciona-se a: quais as tarefas que poderiam ser resolvidas a partir da implementação da tecnologia durante o processo de projeto a fim de evitar futuros transtornos e custos na execução da obra?

Segundo ANDRADE e RUSCHEL (2009) o surgimento de novas tecnologias tem trazido melhoras substanciais para o softwares, como novas funções, novos layouts, integração de informações e dados que podem ser importados e exportados, além de obter também dados por terceiros.

Com a aplicação da tecnologia BIM é possível ter uma melhor visualização da obra, maior produtividade devido ao fácil acesso à informação, maior coordenação dos documentos específicos da construção, integração de informações sobre os elementos e processos de construção, menor tempo de execução e redução de custos.

A base do sistema é um banco de dados que além de definir a geometria dos elementos construtivos em três dimensões, armazena atributos e, portanto, transmite mais informações do que os modelos CAD convencionais. Outra vantagem é que como os elementos são paramétricos, as atualizações e alterações são instantâneas em todo o projeto, facilitando assim, a revisão e aumentando a produtividade. (FLORIO, 2007).

Comparando com outros softwares como o Computer-aided design (CAD), o BIM apresenta vantagens, pois modela e gerencia não somente gráficos, mas também informações que permitem gerar automaticamente desenhos, relatórios, análise de projeto, simulação de cronogramas, facilidade de gerenciamento, entre outras. Assim, a equipe de trabalho pode tomar decisões com base em informações mais precisas e confiáveis. 


\subsection{Panorama geral da implementação da tecnologia BIM}

No século 21, indústria da construção vem crescendo vertiginosamente e esse impulso tem como consequência o surgimento de problemas com a gestão do design, qualidade e eficiência das construções. A competitividade também é outro fator que movimenta o mercado na tentativa de melhorar os serviços prestados ao cliente e à sociedade como um todo (ARANDA-MENA, 2009).

Com o intuito de reduzir esses conflitos, atualmente a tecnologia BIM é vista como uma estratégia para o crescimento sustentado do setor, por melhorar o planejamento e o projeto, reduzir incidência e evitar erros, prazos e desperdícios, além de ampliar a capacidade dos projetistas, otimizar trabalho além do trabalho de quem está no canteiro de obras. No entanto para garantir sucesso e obter todos esses benefícios, é necessário dispor de mão de obra especializada e com nível superior.

Toledo e Barison (2011) consideram fundamental o papel da universidade na formação de recursos humanos devidamente familiarizados com as novas tecnologias do mercado, pois a quantidade de empresas que vem adotando a tecnologia BIM vem crescendo cada vez mais e é preciso preparar os estudantes de nível superior para a realidade do mercado.

Segundo Sacks, Pikas e Hazzan (2013), a indústria da construção precisa de engenheiros de pós-graduação com conhecimentos e habilidades em modelagem de informações (BIM).

Hoje a tecnologia também está sendo utilizada em construções de baixa renda com o incentivo do próprio Governo Federal que vem disponibilizando bibliotecas de componentes BIM para o desenvolvimento do HIS (Habitações de Interesse Social) através do site do Ministério do Desenvolvimento da Indústria e Comércio Exterior (MDIC, 2012).

Paulatinamente, a indústria da construção civil vem se integrando cada vez mais unindo projeto e execução exigindo, assim, profissionais cada vez mais qualificados. No exterior alguns lugares tem nome de destaque na utilização da tecnologia BIM, são eles: Alaska Departamento f Traffic (DOT), California DOT, Florida DOT, Massachusetts DOT, US Army Corporation of Engineers, US Bureu of Land Managements entre outros.

No Brasil, pesquisas publicadas sobre o tema são recentes e em quantidade inexpressiva. Apesar de Manzione (2013) considerar que na atualidade discutir gestão de projeto sem base em TI não tem efeito, ou seja, é inócuo, a maioria das experiências e aplicações do BIM estão ainda em estágio de experimentação. Ainda existe poucos trabalhos e bibliografias que possam comprovar os reais benefícios de seu uso.

\subsection{Tecnologia BIM e gestão de projetos}

$\mathrm{Na}$ indústria imobiliária, as atribuições e responsabilidades da coordenação de projetos deve ser compartilhada e prever, em sua estrutura, o envolvimento de todos os agentes no processo de desenvolvimento do projeto. No entanto, segundo Melhado at al. (2010, p.104) cabe ao coordenador de projetos garantir:

[...] que as soluções técnicas desenvolvidas pelos projetistas de diferentes especialidades sejam congruentes com as necessidades e objetivos do cliente, compatíveis entre si e com a cultura construtiva das empresas construtoras que serão responsáveis pelas respectivas obras.

As principais tarefas que o coordenador de projetos cumpre estão relacionadas com a organização e o planejamento do processo de projeto e à gestão do processo de projeto. 
(MELHADO, 2010).

Melhado (1994) após análise das dificuldades fizando a melhoria da qualidade do processo de projeto das edificações propõe uma "morfologia" desses projetos a fim de obter uma melhor definição das atividades desenvolvidas nas fases que envolve o processo.

Com base nos conceitos referentes à gestão de projetos e o uso da tecnologia BIM Manzione (2013) considera que houve uma grande evolução referente ao projeto denominada Level of Development (LOD). O nível de desenvolvimento com o aprofundamento das questões relacionadas ao projeto oferecido pelo BIM permite que os agentes envolvidos repensem seus modelos de gestão e coordenação estabelecendo, assim, uma maior necessidade de integração dos domínios da tecnologia da informação com os da gestão, reavaliando as fases de projeto e as responsabilidades dos envolvidos em todo o processo.

Nesse contexto Manzione (2013) considera que com a difusão do uso da tecnologia BIM um novo paradigma para trabalhos colaborativos deve ser criado. Outro ponto de vista abordado é que em relação à sua prática e de acordo com a literatura "o BIM terá uma implantação em estágios evolutivos”. (MANZIONE p.36, 2013).

Para Succar (2009) a completa adoção do BIM na indústria da construção não ocorre de forma imediada. Há vários estágios com a apropriação que ocorre de forma gradual da tecnologia e transformação dos processos que estão correlacionados.

A tecnologia do Building Information Modeling (BIM) é um instrumento aplicado no processo de projeto. Muitos pesquisadores de todo o mundo: Austin e Baldwin (1996); Fabrício (2002); Melhado (1994) e Ulrich e Eppinger (1990) estudaram o processo de projeto e em face das necessidades de ampla reformulação a tecnologia BIM e o trabalho colaborativo são considerados essenciais para alcançar essa evolução.

Estudos sobre a gestão do processo de projeto apontam que na prática o planejamento e o controle do processo de projeto convertem-se em situações de caos e improviso o que leva a problemas não apenas na comunicação como também em falhas da geração dos documentos referente ao projeto, erros nas tomadas de decisão gerando situações na maioria das vezes bastante complexas de serem solucionadas.

Para Manzione (p.36, 2013) "as ações para a melhoria do processo estão fortemente influenciadas pela maneira como ele é conceituado e praticado". Assim, acredita que deve-se partir da organização do conhecimento sobre ele.

As edificações são estruturas complexas e que com a evolução do setor de construção, vem produzindo novas situações que permitem práticas colaborativas de trabalho para atender às demandas de mercado. Esse fator impulsiona os profissionais e empresas a buscarem melhores resultados e para isso fazem grandes investimentos em recursos tecnológicos sem necessariamente compreender as mudanças que trarão com a sua adoção. (Manzione, 2013).

Nesse sentido vale ressaltar que a modelagem da informação não deve ser levada como uma "tábua de salvação" como mesmo menciona Manzione (2013), para todos os problemas de uma empresa, o que muitas vezes acontece e o que está sendo abordado no estudo de caso estudado.

\section{ESTUDO DE CASO RESERVA CAMARÁ}

\subsection{Reserva Camará}

Localizado na Região Metropolitana do Recife, a Reserva Camará é um Complexo 
Multiuso, que envolve projetos nas áreas social, cultural e ambiental. Situada numa área de importante cobertura vegetal e diversidade de ecossistemas em 26 hectares, dos quais $30 \%$ estão destinados à preservação da fauna e flora.

O projeto está voltado para atender várias funções dentre elas: habitacional (22 torres residenciais e flats), institucional (universidade), comercial (2 empresariais e um shopping center), como também de serviços e lazer (call center, centro de convenções e museu, sede do Instituto Camará).

O Projeto tem o diferencial de tratar o espaço natural e construído de forma a conscientizar a população de que é possível construir sem destruir, ou seja, visando a redução dos impactos e a conscientização da importância da preservação do meio ambiente.

O Projeto foi desenvolvido com base em 48 conceitos de sustentabilidade, possibilitando com isso a obtenção da certificação LEED ND (Neighborhood Development), principal certificação ambiental para bairros sustentáveis que é reconhecida internacionalmente e desenvolvida pelo US Green Building Council (USGBC).

Além disso, o projeto também conta com a atuação do Grupo Sustentax, consultoria em projetos de sustentabilidade, pioneira na América do Sul nesta atividade de conceber executar empreendimentos imobiliários nos moldes internacionais para novos bairros.

Assim, o projeto Reserva Camará abriga um mix de usos onde os princípios norteadores são os ideais da sustentabilidade abarcando os aspectos: ambiental, social e econômico, na busca de realizar um empreendimento de acordo com os melhores padrões internacionais e também nacionais a exemplo das certificações AQUA e Procel Edifica, desenvolvido por construções isoladas que fazem parte do conjunto do projeto.

\subsection{O Escritório de Coordenação de Projetos}

O escritório de coordenação de projetos funciona dentro da reserva Camará e pertence à empresa incorporadora Reserva Camará. Essa empresa tem um quadro aproximado de 80 funcionários entre funções administrativas, financeiras, jurídicas, marketing e engenharia/obra.

O escritório de coordenação de projetos reúne 4 funcionários: Uma arquiteta sênior coordenadora de projetos, uma engenheira civil, um designer e um estagiário. No projeto da reserva Camará o escritório trabalha com quatro empresas de projetos: Techna (Projetos de instalações complementares), Interplan (climatização), Andrade\&Raposo (arquitetura e urbanismo) e Engedata (projeto de estrutura).

O empreendimento Reserva Camará iniciou suas atividades em janeiro de 2013 e tem prazo final a data de abril de 2015, segundo cronograma abaixo: 
Figura 1 - Cronograma Reserva Camará

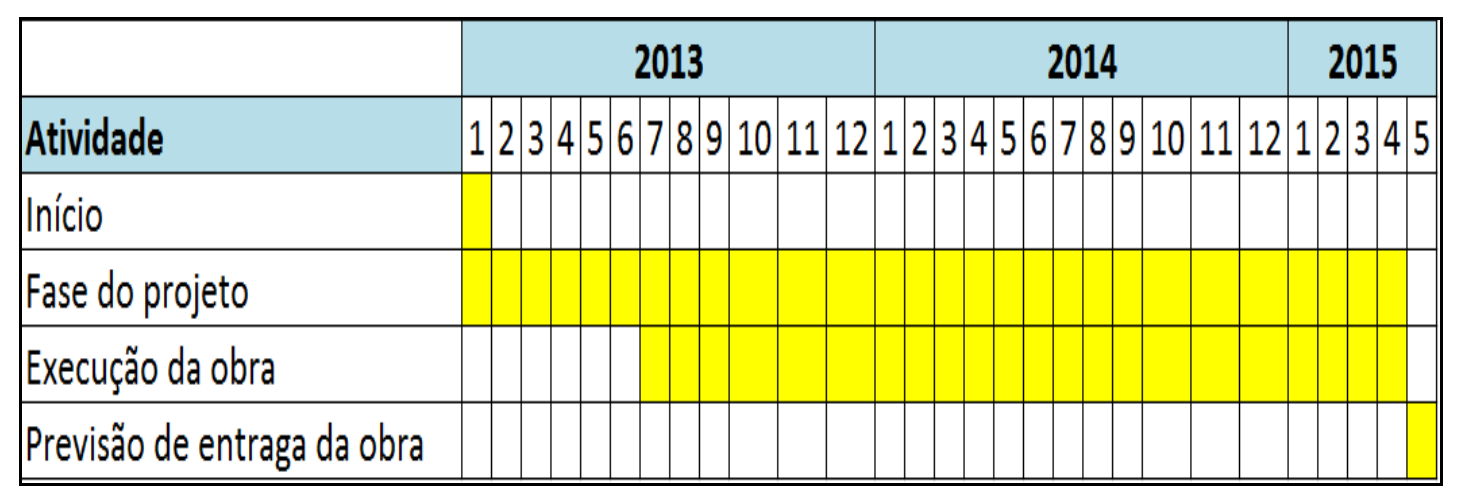

O escritório de coordenação de projetos trabalha com quatro softwares que se complementam entre si: Plataforma Office, AutoCad, Revit, Navisworks, Adobe Premiere Pro. O AutoCad em função de todos os projetos serem recebidos das empresas de projetos nessa plataforma. O Revit é usado para a modelagem BIM, o Navisworks para fazer a análise de projeto, o Adobe Premier Pro para criar e editar os vídeos de simulação 3D.

Desde o início das atividades a empresa de coordenação de projetos, que tinha um engenheiro civil com pouco tempo de formação profissional como seu coordenador e após 5 meses contratou uma arquiteta sênior como coordenadora de projetos, vem trabalhando na análise crítica e compatibilização dos projetos recebidos.

Os projetos são recebidos em formato AutoCad e transformados para modelagem BIM. Após transição para plataforma BIM, com o uso do software Naviworks, começa-se a fazer a análise e compatibilização dos projetos.

Nessa fase, a equipe do escritório de coordenação de projetos começa a levantar as incompatibilidades construtivas dos diversos projetos e a fazer uma análise crítica destes através dos relatórios de erros gerados pelo programa, então se elimina os erros redundantes do processo e se envia um relatório de erros para empresa de projetos. Inicialmente, ao final da análise de cada fase, os erros que eram levantados eram tratados de forma a obedecer dois critérios distintos: Falhas projetuais de maior envergadura eram retornadas para o escritório de projetos para que as alterações fossem procedidas.

Já as falhas projetuais de menor dimensão eram alteradas no próprio escritório de coordenação de projetos e era enviado um relatório constando tais alterações para que o escritório de projetos tivesse conhecimento do que foi alterado. Atualmente, qualquer inconsistência encontrada é relatada para os escritórios de projetos para que sejam corrigidas.

Os fluxogramas abaixo mostram os dois processos, inicial e atual: 


\section{Figura 2 - Fluxograma de Projetos Inicial}

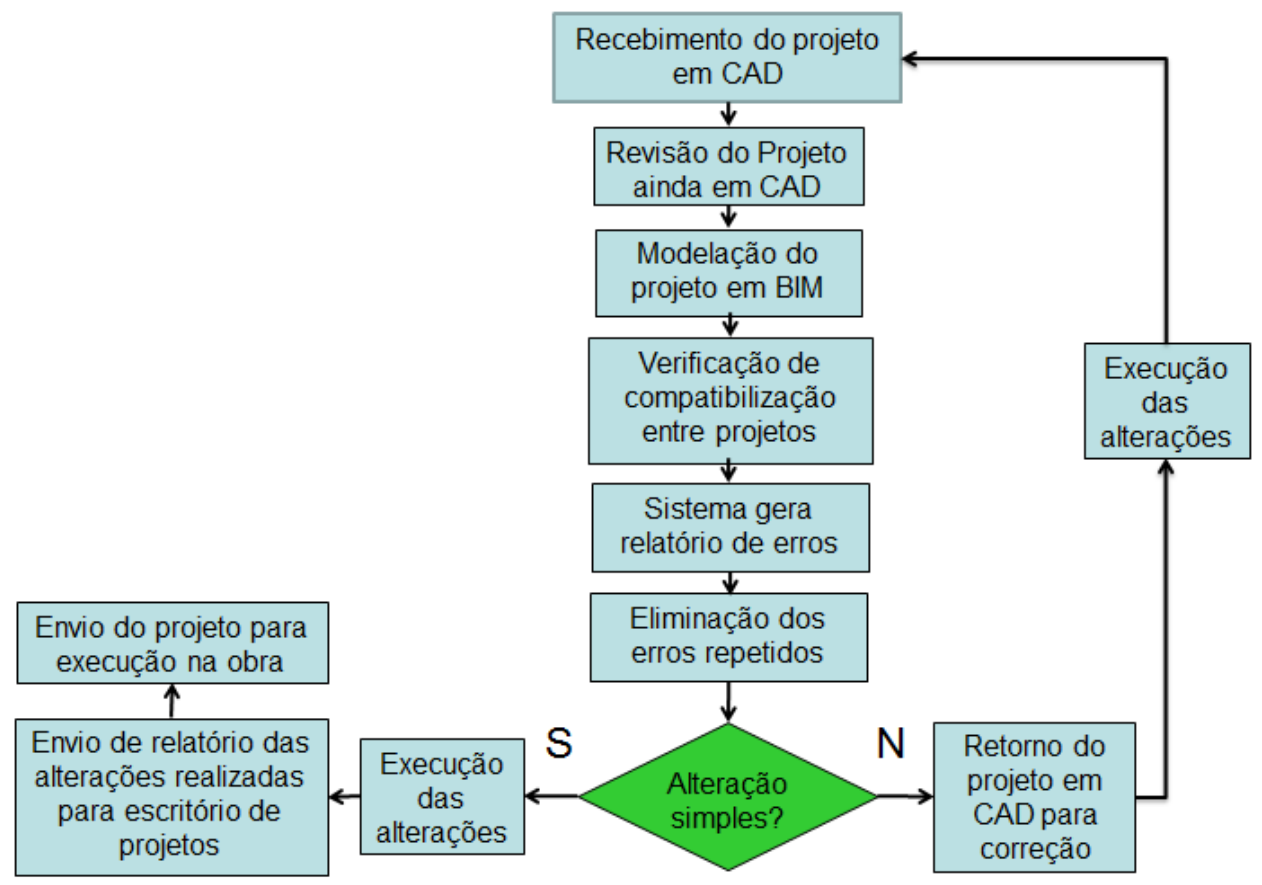

Figura 3 - Fluxograma de Projetos Atual

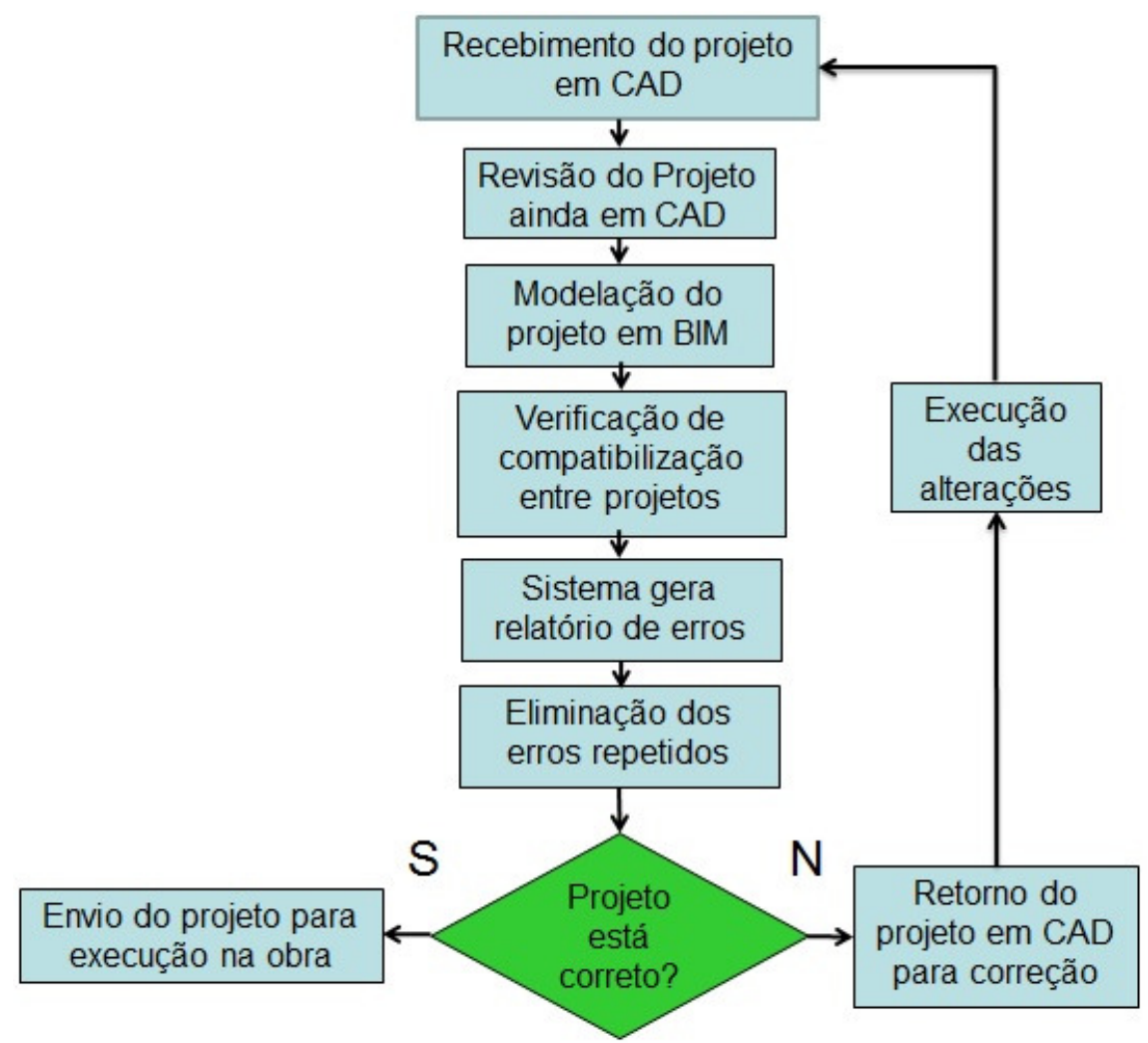

Como o empreendimento ainda está em sua fase inicial, o volume de trabalho não atingiu sua totalidade. Em nível de execução de obra, ele ainda está na fase de fundação. As reuniões com os escritórios de projetos ainda não têm um cronograma definido. 
Essas reuniões têm acontecido sempre que se faz necessário, entretanto, há uma periodicidade média quinzenal, onde são tratados todos os gargalos existentes e esclarecido dúvidas quanto aos projetos.

Algumas dificuldades são relatadas pela equipe em relação a implementação do uso da modelagem BIM. Uma primeira dificuldade levantada é a falta de disponibilidade de modelos ou famílias de modelos BIM no mercado nacional. Pelo fato da plataforma BIM ainda ser uma realidade longe de se tornar popular ou de uso em escala, o mercado nacional ainda carece de modelos eletrônicos de componentes da construção disponíveis na internet.

Com isso, a maioria dos modelos necessários para a correta transferência do padrão CAD para o BIM deve ser desenhada no próprio escritório de coordenação de projetos. Isso toma boa parte do tempo dos desenhistas que precisam criar cada modelo para ser utilizado na plataforma BIM no programa Revit.

Uma outra dificuldade encontrada pela equipe do escritório de projetos tem sido a mão de obra especializada. Por mais que já se tenham no mercado cursos que capacitam profissionais com respeito ao uso do BIM, não se tem profissionais com experiência suficiente para o uso da plataforma em nível de excelência. A opção encontrada foi treinar os próprios estagiários internamente de forma a compor o quadro de desenhistas. Os próprios arquitetos e engenheiros da equipe não têm conhecimento profundo da ferramenta.

Por outro lado, há um ganho muito grande de tempo e redução de custos com a detecção de erros que seriam percebidos somente em fase de execução da obra.

Outro ponto percebido é que não há uma reação negativa das empresas de projeto com o nível de detalhamento ainda em fase de projeto por conta de retorno para correção de inconsistência. Percebe-se um interesse por parte das equipes de projetistas em se aproximar da plataforma BIM, reconhecendo nela mais um aliado que um obstáculo para execução de seus trabalhos. Eles têm encarado essa mudança de paradigma de uma forma propositiva.

\section{CONSIDERAÇÕES FINAIS}

Apesar da implementação da plataforma BIM como meio a se minimizar os custos decorrentes da má compatibilização dos diversos projetos, retrabalho, e, por consequência, aumento de custos por problemas gerados na execução da obra, o processo de receber os projetos no escritório de coordenação de projetos em CAD e transformá-los em um modelo BIM de forma a se melhorar sua gestão, embora venha atendendo as necessidades inicialmente planejadas de implantação da plataforma BIM, além de estar longe de ser a ideal, estabelece uma prática equivocada de trabalho que pode acarretar em uma adoção de um modelo paralelo distorcido.

Faz-se necessário um esforço maior para se implementar um modelo de trabalho onde todo processo, desde a concepção, planejamento e desenho por parte das empresas de projetos até a fase de execução da obra aconteçam utilizando-se da modelagem BIM.

Entende-se que para isso se faz necessário toda uma mudança de paradigma não só de envolvimento de todos os envolvidos no processo de planejamento desenho e execução, mas também num redesenho operacional onde o princípio colaborativo nestas fases entre os seus participantes seja uma premissa para o bom andamento das atividades. 
Segundo Sacks (2012), a tecnologia é interessante e excitante e seu objetivo é criar situações que facilitem as interações entre as diversas equipes, entretanto se o processo colaborativo entre estas equipes não for alcançado, pouco se conseguiu avançar.

Sendo assim, não é apenas mudando a tecnologia que se promoverá uma melhor qualidade do projeto e consequentemente sua execução, mas mudando os procedimentos e fluxos de trabalho de forma a ter a nova plataforma como aliada no processo pela busca da excelência.

\section{REFERÊNCIAS}

ADESSE, Eliana. et al. Manual de Escopo de Serviços para Coordenação de Projetos: Indústria Imobiliária. 2010.São Paulo: Depto. de Engenharia de Construção Civil da Escola Politécnica da USP.

ANDRADE DE, Max Lira; RUSCHEL, Regina Coeli. BIM: Conceitos, cenários das pesquisas publicadas no Brasil e tendências. Anais do Simpósio Brasileiro de Qualidade do Projeto no Ambiente Construído. São Paulo: IX Workshop Brasileiro de Gestão do Processo de Projeto na Construção de Edifícios. 18 a 20 de novembro de 2009. Universidade de São Paulo.

ARANDA-MENA, Guillermo. et al. Building information modelling demystified: does it make business sense to adopt BIM? Vol. 2 No. 3, Vancouver: International Journal of Managing Projects in Business, 2009. pp. 419-433. Emerald Group Publishing Limited 1753-8378 DOI $10.1108 / 17538370910971063$

EASTMAN, C. et al. BIM Handbook: a Guide to Building Information Modeling for Owners, Managers, Designer, Engineers, and Constractor. New Jersey: John Wiley \& Sons, 2008.

FLORIO, W. Contribuições do Building Information Modeling no processo de projeto em arquitetura. Seminário TIC 2007 - Tecnologia da Informação e Comunicação na construção civil, 2007, Porto Alegre: Anais, 2007. TIC 2007 CD ROM.

HAZZAN, O.; PIKAS, E.; SACKS, R. Building Information Modeling Education for Construction Engineering and Management. II: Procedures and Implementation Case Study. Disponível em: http://ascelibrary.org/doi/abs/10.1061/(ASCE)CO.1943-7862.0000765 . Acesso em: novembro 2013.

MANZIONE, Leonardo. Proposição de uma estrutura conceitual de gestão do proceso de projeto colaborativo com o uso do BIM. São Paulo: 2013. 325p. Tese (Doutorado em Engenharia) Departamento de Engenharia de Construção, Escola Politécnica da Universidade de São Paulo.

MELHADO, S.B. Qualidade do projeto na construção de edifícios: aplicação ao caso das empresas de incorporação e construção. São Paulo: 1994. 294p. Tese (Doutorado) - Escola Politécnica, Universidade de São Paulo.

OLIVEIRA, Otávio José de. Modelo de gestão para pequenas empresas de projetos de edificios. São Paulo: 2005. 262f. Tese (Doutorado em Engenharia) - Departamento de Engenharia de Construção Civil, Escola Politécnica da Universidade de São Paulo. 2005. Disponível em: http://pcc5017.pcc.usc.br/Textos\%20PDF/OT\%C381VIO\%20J\%20OLIVEIRA\%20TESE.pdf. Acesso em: setembro 2013.

REVISTA CONSTRUIR NE.Recife: RCN Editores Associados. Agosto 2013. Ed. 68

SACKS, R. Evento do Dia do Gerente de Projetos. Universidade de Tecnologia de Tallinn, 2012.

SUCCAR, B. Building Information Modelling Framework: a research and delivery foundation for industry stakeholders, Automation in Construction, disponível em:

http://www.academia.edu/170356/Building_Information_Modelling_framework_a_research_and_deliver y_foundation_for_industry_stakeholdersv. Acesso em: setembro 2013

TOLEDO, E. S.; BARISON, M. B. Formação de recursos humanos evidamente familiarizados com os novos paradigmas que o BIM pressupõe é essencial e urgente. Revista Construção, v. 115, 2011. 